\title{
Revision del proceso de la administracion estrategica, estudio del paradigma de estrategia, estructura y equifinalidad: una investigacion empirica en empresas en noreste de México (A review of the strategic management process, study of the strategy, structure and equifinality paradigm: an empirical research in northeast Mexico companies)
}

\author{
Aguirre H., O. Flores y V. F. Alatorre \\ UAT, Col. Infonavit, N. Laredo, Tam 88000, México, hquirre@uat.edu.mx, \\ oflores@uat.edu.mx y valatorre@concretostecnicos.com.mx
}

Palabras claves: Caos, estrategia, estructura, equifinalidad, , situacional, sistemas

\begin{abstract}
Resumen. Esta investigación busca comprobar empíricamente el paradigma entre estrategiaestructura y equifinalidad en empresas del noreste de México. Se llevó al cabo un estudio a través de la aplicación de cuestionarios a negocios de esa parte del país. Se consideró como variable independiente la existencia del modelo de Chandler acerca de que la "estructura organizacional sigue a la estrategia". La variable dependiente, se establece como la obtención de resultados equifinales (igualmente finales), con 0 sin la existencia del paradigma de estrategia-estructura. Dos contribuciones emergen de este estudio. La primera consiste en haber encontrado que efectivamente existe una relación entre estrategia y estructura en las empresas investigadas. La segunda, y más importante, es que se evidenció, con prueba empírica, el paradigma de equifinalidad. Se necesita mayor investigación para poder determinar las razones para el funcionamiento de este paradigma. La naturaleza holística de la Administración Estratégica puede ser la que explique el éxito final, "sin importar los inicios".
\end{abstract}

Key words: Chaos, equifinality, situational, strategy, structure, system

\begin{abstract}
The objective of this research is to test empirically the strategy, structure and equifinality paradigm in northeast México companies. Businesses, in this region of the country, were studied through the application of a questionnaire. The paradigm of Chandler regarding "structure follows strategy" is the independent variable. The dependent variable is established as finding equifinal results with or without the presence of the strategy-structure paradigm. From this study, two contributions materialize. The first one is that it is effectively true the relationship between strategy and structure. The second and more important one is that the equifinality paradigm was in fact true and empirical evidence was obtained. To determine the reasons for the functioning of this
\end{abstract}


paradigm, more research is needed. "No matter the beginnings", final success may be explained by the wholistic nature of management.

\section{Introducción}

Esta investigación contribuye a la administración general porque el modelo aquí mostrado y probado, pertenece al acervo cultural de la Teoría de la Organización. El presente escrito puede ser de conveniencia y utilidad para toda aquella persona que desee realizar una investigación en el área de la administración estratégica. El propósito de este trabajo es demostrar empíricamente la hipótesis de que en las empresas mexicanas se presentan el paradigma de estrategia y estructura de Alfred D. Chandler y el principio de equifinalidad de Ludwing von Bertalanffy.

El actual trabajo no pretende penetrar en los conceptos y en las investigaciones que se han escrito acerca de la Teoría de la Organización y la equifinalidad. De la misma manera, no se interesa en ahondar y en explicar profundamente las grandes contribuciones que han hecho los tratadistas y los estudiosos de este tema. Este artículo solo busca ilustrar de una manera práctica y sencilla los modelos ya mencionados acerca de la estrategia, la estructura y la equifinalidad y pretende probar empíricamente el paradigma aludido. La presente investigación está enfocada solamente a cien de las empresas del noreste de este país. Una restricción importante de la misma es que la muestra seleccionada para realizar las encuestas estuvo limitada solamente a esos cien negocios.

\section{Revisión bibliográfica. Síntesis de las diferentes propuestas de modelos de administración estratégica}

Las empresas que siguen el proceso de la administración estratégica tienen mayores probabilidades de tener éxito (Johnson y Scholes, 2001 y Bettis y Prahalad, 1995). De acuerdo con la revisión teórica llevada al cabo, a continuación, se hace una sinopsis de los pasos que se recomiendan en general, deben de seguirse en una empresa, para realizar este proceso de administración estratégica. Se presenta, de esta manera, el sumario que trata de sintetizar el pensamiento estratégico hasta nuestros días (Mintzberg, et al., 1997).

1. Establecimiento de la visión estratégica de la empresa

a. Fijación de los objetivos a largo plazo de la empresa y necesidades sociales que se satisfacen

b. Definición de la misión y de los objetivos del negocio, desde el punto de vista de mercadotecnia, ¿en qué 
negocio estamos?

2. Análisis del medio ambiente interno y externo, a través de las fortalezas, las oportunidades, las debilidades y las amenazas (F.O.D.A.)

a. Ambiente interno

b. Ambiente externo

3. Diseño de las estrategias generales del sistema, decisión de la estructura organizacional, resultados y retroalimentación (Fig. 1).

\section{El paradigma de estrategia estructura de Chandler}

Estrechamente relacionado con la administración estratégica y como una extensión de

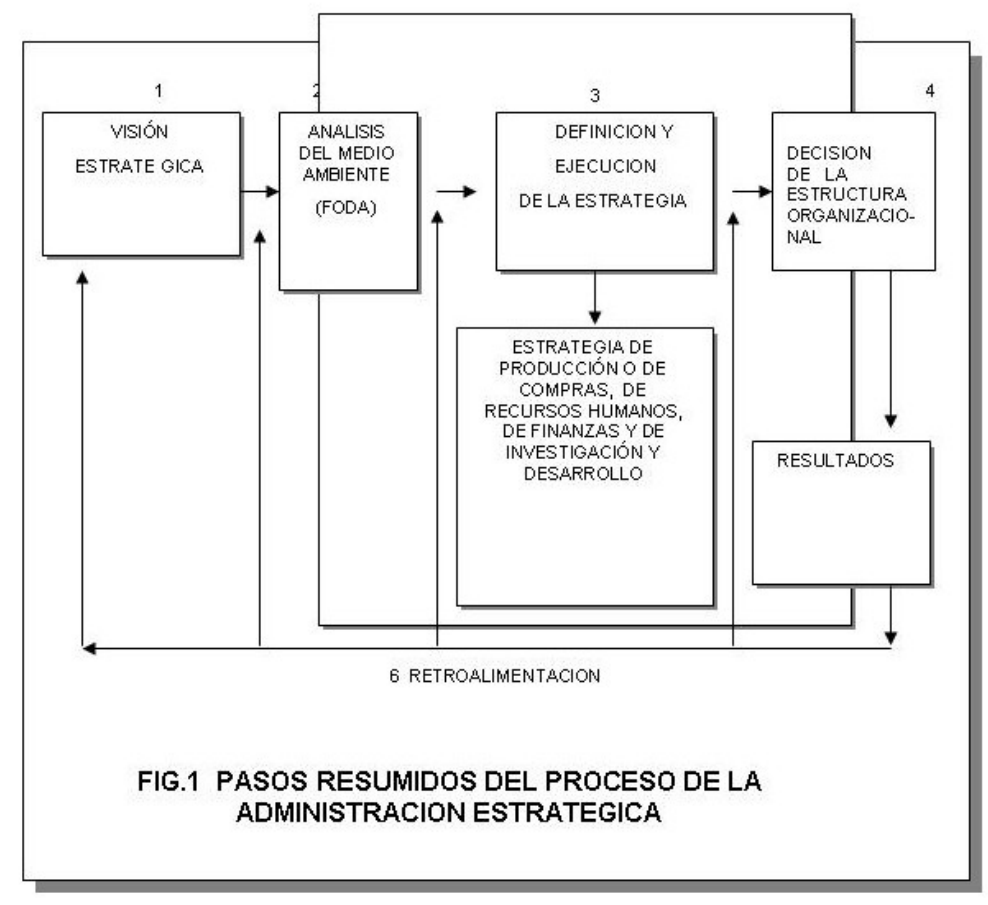

6 RETROALIMENTACION

esta herramienta moderna de gestión, está el modelo de Alfred Chandler de estrategia y estructura. El concepto de estrategia nace de la pregunta: ¿en qué 
negocio está la empresa? Una estrategia "es un conjunto de metas y de políticas principales" como lo define Gary Dessler, en el año 1979. El mencionado autor Alfred Chandler (1962), precisa la estrategia como "la definición de metas básicas y objetivos a largo plazo de la empresa y la adopción de líneas de conducta y la asignación de los recursos necesarios para alcanzar dichas metas".

Agrega Dessler, en este sentido, que las grandes decisiones que lleva al cabo una compañía tales como ampliar el volumen de operaciones, abrir nuevas sucursales e incursionar en nuevas áreas económicas, implican la definición de nuevas metas básicas y la necesidad de una planeación estratégica. Como se analizó en el modelo del Proceso de la Administración Estratégica, presentado anteriormente, existe una interrelación entre la estrategia y la estructura organizacional (diseño departamental) que Alfred Chandler la ha sintetizado en el enunciado siguiente: "la estructura sigue a la estrategia".

Con esta aseveración (Chandler, 1962), quiere significar el vínculo tan estrecho que existe entre la estrategia decidida por la empresa y el tipo de organización o de departamentalización establecida para llevar al cabo la misión estratégica. Reforzando esta idea, señala Dessler, en el año 1979, que para empresas que por la naturaleza de sus actividades, tienen una producción compleja, el contexto es turbulento, se requiere alta tecnología en la producción y el mercado demandante del producto es complicado, será entonces más conveniente poseer una estructura organizacional con una departamentalización divisional (con las modalidades de tipo geográfico, por productos, por proceso 0 por clientes).

Un ejemplo de la anterior situación, se podría presentar hoy en día en los negocios de producción y venta de computadoras, de teléfonos celulares y de otros productos que son de naturaleza complicada, tanto en su fabricación, como en su mercadeo. Esto último, debido a la fuerte competencia de las empresas. Asimismo, la producción que se ofrece al mercado puede ser de productos 0 servicios sencillos y las condiciones circundantes constituyen un medio ambiente tranquilo. Tal es el caso de la fabricación y venta de artículos como serían lápices, sillas y bancos de madera y otros, y de servicios simples que no demanden complejidad en su prestación. En este caso, Chandler recomendaría una estructura organizacional de tipo funcional o también conocida como de procesos funcionales (Dessler, 1979).

Desde 1962, que Alfred Chandler propuso el mencionado paradigma de estrategia-estructura, varios escritos importantes se han desarrollado alrededor de este tema. Señala Miller (1987) que los gerentes tienen una fuerte y especial opinión acerca de la combinación entre la estrategia y la estructura que será conveniente para una empresa determinada. En algunas ocasiones van a buscar una negociación entre varias mezclas. 
Por ejemplo, pueden encontrar una estructura optativa que cumpla igualmente con la función o implementarán otra configuración de acuerdo a preferencias personales y subjetivas 0 utilizaran alguna diferente de la cual tengan la experiencia de que ya ha funcionado antes. Continuando con el tema de las configuraciones organizacionales, Mintzberg $(1979,1993)$, propuso el Modelo Pentagón de las Estructuras Organizacionales Ideales en donde describe, analiza y propone los diferentes tipos "ideales de diseños organizacionales". Su teoría ha sido muy popular por varios años y ha motivado análisis críticos como los que se explican más adelante en esta revisión bibliográfica.

Este autor formula el paradigma mencionado como un modelo de "cinco esquinas" en donde cada una de ellas representa una "estructura organizacional ideal". Estas son: estructura smple, burocracia alrededor de la maquinaria, burocracia profesional, estructura divisional y adhocracia. Establece este autor que cada uno de los diseños propuestos, se considera que "encaja" o se "adapta" a ciertos factores circunstanciales o del contexto. Estos parámetros son el tamaño de la empresa, el medio ambiente, su volatibilidad, la tecnología y otros.

Esta teoría de las cinco configuraciones ideales ha sido reconocida por los intelectuales de la Teoría Organizacional, pero padece de suficiente prueba empírica. Doty et al. (1993), proveen una adecuada revisión de esta investigación, proponiendo como una extensión del modelo, que cada una de las estructuras ideales de Mintzberg debe de asociarse precisamente con un medio ambiente concreto y único. Le la calidad de este embone adecuado entre la estructura y las condiciones circundantes, dependerá la efectividad del proceso. Un análisis de éste y una crítica del mismo, se presenta a continuación por William A. Drago en 1998.

El mencionado autor, Drago (1998), llevó al cabo una investigación para probar la mencionada teoría de Mintzberg a través de obtener información de ejecutivos de 91 empresas. Los cuestionarios buscaban identificar relaciones entre estrategia, estructura, medio ambiente y rendimiento. Las empresas fueron seleccionadas aleatoriamente del listado de empresas conocido como Standard and Poor's Industrial Compustat. Los cuestionarios fueron enviados por correo directamente a los ejecutivos. A éstos se les pidió que identificaran ciertas características estructurales de sus organizaciones y las relacionaran con los cinco tipos ideales de Mintzberg. A continuación se presentan las estructuras ideales propuestas por el autor y las características correspondidas con cada estructura (también llamadas "índices" por Mintzberg), encontradas por Drago (1998), en su investigación:

Primer tipo ideal: Estructura simple 
Características:

A. La supervisión directa es un mecanismo coordinador primario en las operaciones.

B. La toma de decisiones es guiada por un fuerte sentido de la misiónvisión.

C. La comunicación es vertical y sigue la jerarquía de la gerencia.

D. La comunicación se lleva al cabo a través de líneas formales.

E. La toma de decisiones se realiza siguiendo los procedimientos operativos estándar.

Segundo tipo ideal: Burocracia alrededor de la maquinaria

\section{Características:}

A. La estandarización de tareas en las operaciones es un mecanismo coordinador primario.

B. La toma de decisiones se lleva al cabo por procedimientos operativos estándar.

C. La comunicación se estructura de manera formal.

D. La toma de decisiones se realiza por un plan programado de actividades.

E. La comunicación es vertical, siguiendo la jerarquía.

F. La organización sigue un criterio de departamentalización funcional

(el organigrama es con base a las funciones principales de la empresa).

Tercer tipo ideal: Estructura divisional

\section{Caracteristicas:}

A. La estandarización de los resultados es un mecanismo coordinador primario.

B. La comunicación es autónoma y vertical. Esta sigue la jerarquía de la administración.

C. La comunicación se regula a través de líneas formales.

D. Las unidades principales están organizadas por departamentalización por productos.

E. Los departamentos más importantes actúan en forma autónoma.

F. Los departamentos más importantes disponen de un servicio de asesoría de expertos.

Cuarto tipo ideal: Adhocracia.

\section{Características:}

A. La comunicación es horizontal al pasar de una actividad a la otra.

B. Los empleados tienen una gran influencia en las decisiones estratégicas.

C. La supervisión directa es un mecanismo muy importante de 
coordinación en las operaciones.

D. La estandardización de tareas es un mecanismo muy importante en las operaciones.

E. La comunicación es regulada a través de conductos formales.

F. La toma de decisiones es por procedimientos operativos estándar.

\section{Quinto tipo ideal: Estructura profesional}

\section{Características:}

A. La estandarización de las habilidades es un mecanismo coordinador muy importante en las operaciones.

B. Los empleados tienen una influencia muy significativa en las decisiones estratégicas.

C. Los departamentos más importantes actúan de manera autónoma.

D. Las habilidades de los empleados constituyen un recurso importante para la organización.

E. La comunicación es horizontal de una actividad a la otra.

F. La supervisión directa es un mecanismo coordinador muy importante en la organización.

Drago concluye que la mayoría de las organizaciones estudiadas "encaja" dentro de las estructuras ideales de Mintzberg. Es más común (tres a uno) encontrar las estructuras ideales que otras diferentes que Drago menciona como híbridas. La adhocracia es una forma popular y repetitiva en las empresas encuestadas. También se encontraron adhocracias en los negocios denominados de naturaleza híbrida.

Las aseveraciones de Mintzberg fueron confirmadas en esta investigación, pero también se encontraron situaciones que no corroboran estas afirmaciones y disgregan en varios casos. Por ejemplo, en las organizaciones en la categoría de estructuras simples, se localizó alta volatilidad en el contexto 0 medio ambiente. Además se halló que estas organizaciones sencillas son relativamente diversas. Las organizaciones (o burocracias) edificadas alrededor de la maquinaria, concluyó Drago, tienen pocos empleados, diferente a lo planteado por Mintzberg en esa particular "estructura ideal".

De los anteriores cinco tipos de organizaciones, posiblemente el más novedoso e interesante sea la adhocracia. Este concepto merece un poco más de atención. La adhocracia es un tipo de organización con una estructura complicada y estandarizada. Es voluble y la autoridad se cambia constantemente. El control y la coordinación se llevan al cabo a través de un ajuste mutuo, por medio de las comunicaciones informales y la interacción entre los ejecutivos. 
Las bases de apoyo de este tipo de estructura son los expertos entrenados y especializados para realizar la mayor parte de las actividades que se llevan al cabo de manera plural. El compromiso común de todos los que colaboran en este tipo de organización es muy importante y motiva la coordinación entre ellos mediante mecanismos de unión.

En la adhocracia los expertos están diseminados por toda la organización y las órdenes están distribuidas dependiendo de la circunstancia 0 del estado de avance del trabajo, ya que depende de la ubicación del experto que en cada situación se requiera para tomar una decisión determinada. Existen muchos gerentes en la adhocracia, pero no ejercen la supervisión de manera clásica, sino más bien están preocupados de la integración de los diferentes grupos. La estrategia no fluye desde lo alto, sino más bien es desarrollada de acuerdo a la toma de decisiones específica en cada proyecto; o sea, la estrategia se genera y se acepta a medida que se crean y se desarrollan nuevos proyectos.

Algunos ejemplos de este tipo de organizaciones pueden ser las agencias de creatividad de mercadotecnia y las empresas consultoras de estrategias, donde cada ejecutivo es un experto del área que se ocupa, siendo importante la buena relación y apoyo entre las partes. [En Red] (http://www.uned.es).

Adhocracia es un neologismo y consiste en una estructura que está en oposición a la burocracia. Es un término utilizado por Alvin Toffler al referirse a un concepto opuesto por naturaleza a la burocracia. Esta última se maneja con rutinas, mientras que la adhocracia acorta y simplifica los procesos, adaptándose a cada situación particular. La palabra es una derivación de ad hoc , que significa "para un fin determinado" [En Red] (http://www.deguate.com).

El modelo situacional, que será analizado más adelante, en esta revisión bibliográfica, aplicado al paradigma de Chandler, básicamente sugiere que un acomodamiento determinado de estrategia-estructura conviene a un tipo específico de ambiente de una compañía. Para otro contexto empresarial, una relación diferente de estrategia-estructura será el indicado (Lorsch y Morse, 1974). Otros investigadores de temas relacionados con el paradigma de estrategia y estructura como lo son Hage y Aiken, en 1969 han sugerido que una relación específica entre estrategia y estructura convendrá para una industria determinada por las características de su medio ambiente.

El paradigma de Alfred Chandler ofrece la base para partir hacia nuevas suposiciones que son analizadas en este trabajo y probadas en el campo. Esto se seguirá comentando más adelante, cuando se explore el enfoque situacional, que constituye el sustento filosófico de la propuesta de esta disertación. 


\section{El enfoque de sistemas}

El biólogo y físico Ludwig von Bertalanffy, hace cerca de 75 años, inició varios estudios para investigar el movimiento de los organismos vivos dentro de un sistema biológico, caracterizándolos como sistemas abiertos y diferenciándolos de los sistemas cerrados. Este científico es reconocido como fundador de la Teoría General de Sistemas (Certo, 2001).

La concepción de "la totalidad" es inherente a la de sistemas. L. Thomas Hopkins, en 1937 (citado en Wechsler, 1998), propone los siguientes pasos para llevar al cabo un análisis de sistemas:

1. Debe de dársele mayor importancia al todo; las partes, tienen un valor secundario.

2. La interrelación de los elementos con el todo logra la integración de un sistema.

3. La modificación de una de sus partes se debe de ponderar si afecta a otra parte.

4. Para que el todo pueda lograr su objetivo, cada parte del sistema necesita desempeñar una función.

5. La ubicación y el lugar de una parte en el todo se define por la naturaleza de ella y de su función.

6. Todos los elementos interrelacionados evolucionan para adecuarse mejor al objetivo de la totalidad.

Continuando el principio de totalidad de los sistemas, el investigador Ludwig von Bertalanffy señalaba, como principal proposición de su modelo, que para comprender íntegramente la operación de una entidad, ésta debe de ser analizada como un sistema. Asimismo, instituyó el concepto de "equifinalidad", estableciendo que se logra un mismo estado final a través de diferentes formas y con condiciones iniciales diversas (von Bertalanffy, 1930, 1960).

De todos los anteriores conceptos, nace el Enfoque de Sistemas aplicado a las organizaciones. Parte de la base de que en lugar de estudiar y de tratar a los diversos segmentos de la empresa como entes separados, considera que la organización es un sistema integral que tiene un objetivo y está formado por subsistemas que se interrelacionan entre si.

\section{La teoría del caos}


La Teoría del Caos es el estudio de patrones dinámicos dentro de los sistemas sociales amplios (Stoner et al., 1996). En los últimos años, dentro de las investigaciones de la Teoría de la Organización, ha existido un renacido interés por el análisis del Caos. El área que lo comprende es conocida como la Ciencia de la Complejidad. Algunos logros recientes y aislados se han estado dando en el área de la administración, sobre todo en mercadotecnia (Deshpande y Farley, 1998).

El paradigma de la Teoría del Caos ha tenido una profunda influencia en las ciencias físicas y en las ciencias administrativas. Los paradigmas que explican hasta la fecha, el comportamiento del liderazgo, por ejemplo, deberán pronto ir cambiando. Desafortunadamente, el lenguaje en que está escrito este tema es muy complejo y rebuscado y solo pocos, buenos documentos, existen relacionados con el Caos aplicado a la organización (Burns, 2002).

Usualmente los teóricos de la escuela de sistemas se dividen entre los que sostienen una posición mecanicista o rigorista y una posición vitalista $u$ organicista. La Teoría del Caos es una nueva ciencia y pretende unir "o traer a un solo cuerpo de principios" a los dos enfoques, el mecanicista y el organicista (Gurpreet y Ward, 2002). Otros autores puntualizan la aplicabilidad de la Teoría del Caos a los fenómenos de la organización en donde el comportamiento de las personas, las resultantes de su actuación y las interacciones con el contexto no pueden ser analizados en un sentido lineal y estricto (Stacey, 1991).

La Teoría del Caos "cruza o atraviesa" las líneas que separan a las disciplinas científicas. Constituye una ciencia de la naturaleza global de los sistemas y ha estado atrayendo a diversos pensadores que usualmente han estado en campos de estudio separados (Peters, 1991). En los estudios sobre organización es común que se establezca que es conveniente la fijación de objetivos a largo plazo y continuamente se hacen pronósticos de resultados futuros. La Teoría del Caos reconoce que los planes a largo plazo, los pronósticos económicos y la visualización de metas al futuro, tienen escasos beneficios para una empresa, son de uso extremadamente limitado y pueden llevar a tener un falso sentido de seguridad.

Para el anterior caso, es común en el ejecutivo, oír que diga: "no debemos de preocuparnos, poseemos un plan para prácticamente cada contingencia". La Teoría del Caos nos enseña que el éxito a largo plazo está en aferrarse al propósito de la compañía y a los valores del negocio (Burns, 2002).

Algunos autores, entre ellos Hock (1996), han señalado que a través de "las lentes" del

Caos (así abrevian los investigadores a la Teoría del Caos), se perciben a las organizaciones como complejas y dinámicas, constituyendo sistemas no lineales en donde el caos y el orden co existen. Estas interrelaciones se expresan en la 
noción de "sistemas caórdicos" o simplemente "caordes", términos acuñados por ese autor.

Este investigador continúa filosofando acerca de la Teoría del Caos, estableciendo que una organización caórdica es una institución que está fuera de control y en orden. Si algo puede estar simultáneamente ordenado y caótico, diseñado de tal manera que no domina el orden o el caos y existe en una fase en medio de ambos, entonces será un ente caótico [En Red] www.cyberspace.com.

De alguna manera, la Teoría del Caos (también llamada Teoría de la Complejidad, por algunos investigadores), se funda en los principios de la Teoría de los Sistemas, partiendo de la base de que éstos están compuestos de partes interrelacionadas 0 agentes interdependientes los cuales obedecen a un conjunto de reglas de comportamiento que son sencillas y comunes.

Algunos autores son indiferentes en cuanto a referirse al anterior fenómeno, tanto como Teoría del Caos, o como Teoría de la Complejidad. Sin embargo, Chennai (2003), señala las diferencias entre ambas teorías. La Teoría del Caos estudia esencialmente los efectos no lineales de los sistemas determinísticos.

Estos últimos son los investigados por la física clásica en donde se conoce el posible resultado o el camino más común que probablemente tome un conjunto de fenómenos. La Teoría de la Complejidad, a comparación, analiza los diseños definitivos de los sistemas que no son determinísticos. El investigador mencionado ilustra con un ejemplo, las diferencias de ambos modelos.

Establece que si estudiamos el movimiento del péndulo, por ejemplo, ya sabemos, que una vez que lo empujamos, hacia que dirección particular se va a mover, pudiendo percibir los movimientos que seguirán, o la tendencia de ellos, los cuales se repetirán. Si perturbamos el péndulo, con un pequeño golpe, no será posible predecir la dirección específica que el péndulo tomará. Este sistema determinístico lo puede estudiar la Teoría del Caos.

La Teoría de la Complejidad es complementaria a la del Caos. Un sistema altamente incierto como un terremoto ilustra la aplicación de la Teoría de la Complejidad. Las investigaciones señalan que existen diseños 0 configuraciones de posibles fenómenos de este tipo para una región en particular. Estos "mapeos" posibles puede ser estudiados por la Teoría de la Complejidad, dado la naturaleza no determinística del fenómeno. El mercado de valores ofrece otro ejemplo parecido al anterior (Chennai, 2003).

La perspectiva de todo lo anterior, acerca de la Teoría del Caos, en el enfoque de Sistemas, y de su impacto y aplicaciones en la organización, ha sido estudiado por Kauffman en el año 1995 en su libro: Laws of Self-Organization and Complexity. El origen de la Teoría del Caos puede ser atribuido a 
Kovalevskaya en 1889 cuando analizando lo cambiante de algunos sistemas, propuso la definición matemática de la inestabilidad dinámica (DeGrauwe, et al., 1993).

En 1920, Julia y Fatou (citado por Gurpreet y Ward, 2002), dos matemáticos, utilizaron el concepto novedoso para estudiar algunas formas geométricas poco usuales. En los setentas esto llevó a Mandelbrot a generar la Teoría de los Fractales Caóticos, como una aplicación de la Teoría del Caos, para analizar los precios del algodón que se habían comportado históricame nte de una manera aleatoria.

Esto tuvo como beneficio el que se identificaran patrones de cambio en los precios, que se repetían en diferentes periodos de tiempo. Posteriormente la Teoría del Caos se ha utilizado para analizar el comportamiento de las acciones y las obligaciones en la bolsa de valores (Radzicki, 1990).

En el siglo diecinueve, los físicos de la época empezaron a explorar las ecuaciones y teorías del paradigma de Newton. Fue en 1961 que un meteorólogo y matemático llamado Edward Lorenz, accidentalmente desarrolló las relaciones y ecuaciones iniciales que revelaron el nacimiento formal de la nueva Teoría del Caos (Gleick, 1987).

En los últimos años, ha estado aumentando la velocidad con la que se dan los cambios en el medio ambiente y en la empresa en particular. La cantidad de información en el mundo está disponible cada vez más rápido y se está doblando cada cinco años (Prichet y Pound, 2001).

\section{La escuela situacional}

El enfoque situacional de la administración parte de la øncepción de que cada circunstancia en la vida de la compañía es diferente y por lo tanto se requiere una manera distinta de dirigir para cada caso diferente (Dessler, 1979). Cada empresa tiene diferentes contextos y situaciones diferentes lo que determina que se tenga que llevar al cabo un proceso de adaptación a las circunstancias específicas (Galbraith, 1973).

En la filosofía situacional, también llamada Teoría de Contingencias, se establece que la efectividad organizacional es la resultante de la sabiduría para diagnosticar, y posteriormente adaptar la manera de administrar, a las características propias de la empresa (Burns y Stalker, 1961). La Escuela de Contingencias en el estudio de la administración de las empresas hace énfasis en que lo que los directivos llevan al cabo en la práctica, depende de un conjunto de circunstancias de una situación determinada (Certo, 2001).

Una vez hecho el diagnóstico de la situación o de las condiciones que rodean (circundantes) a la empresa, se procede a analizar la situación 
interna de la empresa y a tratar de adaptarla a ese medio ambiente externo (Pugh et al., 1969). Estas particularidades, a las cuales debe adaptarse la dirección de la empresa, serán la naturaleza del ambiente de trabajo, como lo establecen Burns y Stalker, en 1961, y la estrategia específica, como lo indica Chandler en el año 1962.

Venkatraman y Prescott (1990), han estado interesados y han llevado al cabo investigaciones sobre la situacionalidad y la "naturaleza negociada" que existe entre las diferentes posibilidades de diversas configuraciones departamentales en relación al medio ambiente. Dependiendo de las condiciones circundantes y de las contingencias especiales que viva el sistema de trabajo en un momento determinado, será la comb inación específica entre la estrategia que decida la empresa y la forma en que ésta deberá organizarse (Dill, 1958).

Meyer, et al., en el año 1993, han señalado, que la importancia del contexto en relación con las decisiones sobre estructuras organizacionales, siempre ha estado presente en los escritos de los pensadores sobre la teoría administrativa. El estudio del "acomodo" entre las configuraciones departamentales y las características de las condiciones circundantes ha sido siempre un reto para los intelectuales del campo de la organización (Lawrence y Lorsch, 1969).

\section{El concepto de equifinalidad}

Equifinalidad, es una noción propuesta por Ludwig von Bertalanffy en 1930 y es la propiedad de los sistemas abiertos para alcanzar un resultado igual, partiendo de condiciones iniciales diferentes y por disímiles maneras. En otras palabras, equifinalidad es lograr "un final igual" (von Bertalanffy, 1968).

Después de los estudios de Bertalanffy, varios investigadores en el campo de la Teoría Organizacional han hecho contribuciones importantes en el terreno de la administración. Los primeros autores que pueden ser incluidos en una revisión teórica son Katz y Kahn por sus investigaciones en los sesentas sobre Teoría Organizacional. Estos autores de las organizaciones en 1966 analizaron las propiedades de los sistemas abiertos e incluyeron la noción de equifinalidad.

El Enfoque de Sistemas (Escuela Sistemática o Corriente Sistémica), el cual incluía los conceptos antes mencionados, tuvo un gran auge al principio de la década de los setentas. Sin embargo, a partir de 1976 fue quedando fuera de moda (Ashmos y Huber, 1987). Sin embargo, varios autores emprendieron la investigación del concepto de equifinalidad, relacionándolo con el estudio de la Teoría de las Organizaciones. 
Las relaciones de los ambientes organizacionales con los conceptos de sistemas, de equifinalidad, de homeostasis y de sistemas abiertos y cerrados, fue robustecida por Katz y Kahn (1978) en la década de los setentas. Posteriormente, se desarrollaron teorías en donde se establecía que el éxito empresarial (0 "resultado final" para el principio de equifinalidad) se puede lograr a través de diferentes estructuras organizacionales, aún si las contingencias a las que se enfrenta el sistema son las mismas (Hrebeniak y Joyce, 1985).

Partiendo del anterior supuesto de Katz y Kahn en el sentido de que un sistema puede alcanzar el mismo nivel final de efectividad organizacional a través de una multitud de senderos y partiendo de una variedad de inicios, Van de Ven y Drazin (1985) y Nadler y Tushman (1988), establecen que esto debe permitir la validez de la existencia de un conjunto de mezclas de diseños de estructuras y contextos empresariales, igualmente efectivos e internamente consistentes. De igual manera lo señala Scott, en el año de 1992.

Algunos estudiosos como Gresov y Drazin en 1997 han establecido que la noción de equifinalidad puede relacionarse con el argumento de superioridad e inferioridad de las estrategias. Asimismo, Jennings y Seaman han realizado estudios en este sentido en el año 1994.

También Matsuno y Mentzer han considerado algo similar en el año 2000 , identificando la equifinalidad con el proceso de llevar al cabo las estrategias de una empresa. Aplicados estos conceptos a la Administración Estratégica actual, se puede concluir que en la mayoría de las empresas no existe una estrategia superior a otra. Porter (1980) y Miles y Snow (1978) así lo señalan en estudios al respecto.

Es importante hacer notar que dentro de la mayoría de los estudios que existen acerca de mercadotecnia y de administración estratégica, diversos investigadores coinciden con la aplicabilidad del concepto de equifinalidad al concluir que no importando las diferentes 0 muy variadas estrategias que se emprendan, la resultante final puede ser la misma (Miles et al., 1978 ; Van de Ven y Drazin, 1985).

\section{Modelo teórico e hipótesis}

El planteamiento del problema se integra de la siguiente manera: se desea investigar el problema que constituye saber si el paradigma equifinalidad se cumple en la administración de empresas del noreste de México.

Los anteriores escritos sobre el proceso de la Administración Estratégica, los conceptos de sistemas abiertos y de sistemas cerrados, el Enfoque de Contingencias y el modelo de von Bertalanffy acerca de la equifinalidad, revisados en este estudio, sirven como base del conocimiento para 
plantear la hipótesis en este documento. A continuación se presenta ésta: "El paradigma acerca de equifinalidad es válido para la administración de empresas en el noreste de México". Esta hipótesis direccional puede ser establecida también de la siguiente manera: "El desempeño de las ventas no depende de las características de complejidad o sencillez, ni del tipo de estructura organizacional de la empresa".

\section{Metodología}

Siguiendo el método analítico, se desglosaron las principales aportaciones de los tratadistas en el área de este estudio. La metodología analógica fue utilizada para comparar conceptos y ubicar más eficientemente los paradigmas de los diferentes autores, que a lo largo de los últimos años, han escrito sobre el tema que ocupa a esta tesis. También se emplearon la inducción, el método deductivo y el sintético.

Para llevar al cabo el presente estudio, se elaboró un cuestionario que se aplicó a un total de 100 empresas en Nuevo Laredo, Cd. Miguel Alemán, Reynosa y Matamoros en el estado de Tamaulipas, en Piedras Negras y Acuña en el estado de Coahuila y en Monterrey, en el estado de Nuevo León.

A través de siete interrogantes se buscó obtener información relevante acerca de los siguientes rubros: giro empresarial, número de empleados, ventas anuales, antigüedad, estructura organizacional (tipo de departamentalización: funcional, divisional o matricial), complejidad de la estrategia y evaluación financiera (ventas).

Los datos de los resultados que arrojó la investigación se analizaron con el programa SPSS. Posteriormente se procedió al examen de la información el cual se presenta a continuación. En resumen, la metodología giró alrededor de investigar la relación entre dos variables. La primera se centra en la existencia del modelo de Chandler acerca de que la estructura organizacional sigue a la estrategia. La segunda, la variable dependiente, se establece como la obtención de resultados equifinales (igualmente finales), con 0 sin la existencia del paradigma de estrategia-estructura.

\section{Análisis datos estadísticos de las pruebas de hipótesis}

\section{- Paradigma de Chandler}

Se establecieron las siguientes hipótesis nula y alterna, Ho: estructura organizacional y complejidad son independientes y Ha: estructura organizacional 
60

y complejidad son dependientes. La prueba empírica se llevó al cabo, por una parte, a través del análisis de la prueba de dependencia chi-cuadrada, entre la estructura organizacional y la complejidad, (ver tabla 1).

Se encontró que el nivel de significancia es menor a 0.001, por lo tanto rechazamos la hipótesis nula. Por consiguiente, la estructura organizacional depende de la complejidad. Entonces podemos concluir que el paradigma de Chandler si se aplica a la manera en que se encuentran organizadas las empresas que participaron en este estudio.

\section{Análisis de varianza para la variable ventas}

Además de la prueba chi-cuadrada, se llevó al cabo el análisis de varianza, para la variable ventas, planteándose las siguientes hipótesis:

Hipótesis nula no. 1 Ho1: No hay diferencia en el desempeño de las empresas, respecto a las ventas, considerando el factor estructura organizacional.

Hipótesis alterna no. 1 Ha1: Si hay diferencia en el desempeño de las empresas, respecto a las ventas, considerando el factor estructura organizacional.

Hipótesis nula no. 2 Ho2: No hay diferencia en el desempeño de las empresas, respecto a las ventas, considerando el factor complejidad.

Hipótesis alterna no. 2 Ha2: Si hay diferencia en el desempeño de las empresas, respecto a las ventas, considerando el factor complejidad.

Hipótesis nula no. 3 Ho3: No existe interacción entre estructura organizacional y complejidad.

Hipótesis alterna no. 3 Ha3: Si existe interacción entre estructura organizacional y complejidad.

Se concluye que no se rechaza la hipótesis nula Ho1 para la estructura organizacional (X5), cuyo nivel de significancia en esa tabla es de .582. Esto comprueba que no hay diferencia en el desempeño de las empresas, respecto a las ventas, considerando el factor estructura organizacional. Respecto a Ho2 para la complejidad ( $\left.X_{6}\right)$, esta hipótesis nula no se rechaza pues el nivel de significancia es de .078 y evidencia que no hay diferencia en el desempeño de las empresas, respecto a las ventas, considerando el factor complejidad.

Con respecto a la interacción de la estructura organizacional y la complejidad, su nivel de significancia es de 0.867 , por lo tanto, no rechazamos la hipótesis nula Ho3. Esto indica que no existe efecto de interacción entre estructura organizacional y complejidad en el análisis de las ventas. El análisis de la varianza se presenta en la tabla 2. 
Tabla 1. resumen de prueba de dependencia chi-cuadrada (a1 cells (16.7\%) have ex pected count less than 5 . The minimum expected count is 3.33.).

\begin{tabular}{|c|c|c|c|c|c|c|}
\hline & Casos & & & & & \\
\hline & $\begin{array}{c}\text { Valid } \\
\mathrm{N}\end{array}$ & Percent & Missing & Percent & $\begin{array}{c}\text { Total } \\
\mathrm{N}\end{array}$ & Percent \\
\hline $\begin{array}{c}\text { Estructura Org.* } \\
\text { Complejidad }\end{array}$ & 100 & $100.0 \%$ & 0 & $.0 \%$ & 100 & $100.0 \%$ \\
\hline
\end{tabular}

Cruzada

Estructura Org * Complejidad Tabulación

Cuenta

$$
\text { Complejidad Total }
$$

Sencillo Complejo

$\begin{array}{ccccc}\text { Estructura Org. } & \text { Funcional } & 55 & 6 & 61 \\ & \text { Divisional } & 8 & 22 & 30 \\ & \text { Matricial } & & 9 & 9 \\ \text { Total } & & 63 & 37 & 100\end{array}$

\begin{tabular}{|c|c|c|c|}
\hline & \multicolumn{3}{|c|}{ Pueba Chi-Cuadrada } \\
\hline & Valor & df & $\begin{array}{c}\text { Asymp. Sig. (2- } \\
\text { sided)(significancia) }\end{array}$ \\
\hline Pearson Chi-Cuad. & 51.624 & 2 & .000 \\
\hline Likelihood Ratio & 57.777 & 2 & .000 \\
\hline $\begin{array}{l}\text { Linear-by-Linear } \\
\text { Association }\end{array}$ & 48.899 & 1 & .000 \\
\hline No. Casos Validos & 100 & & \\
\hline
\end{tabular}


62

Tabla 2. Analisis de varianza.

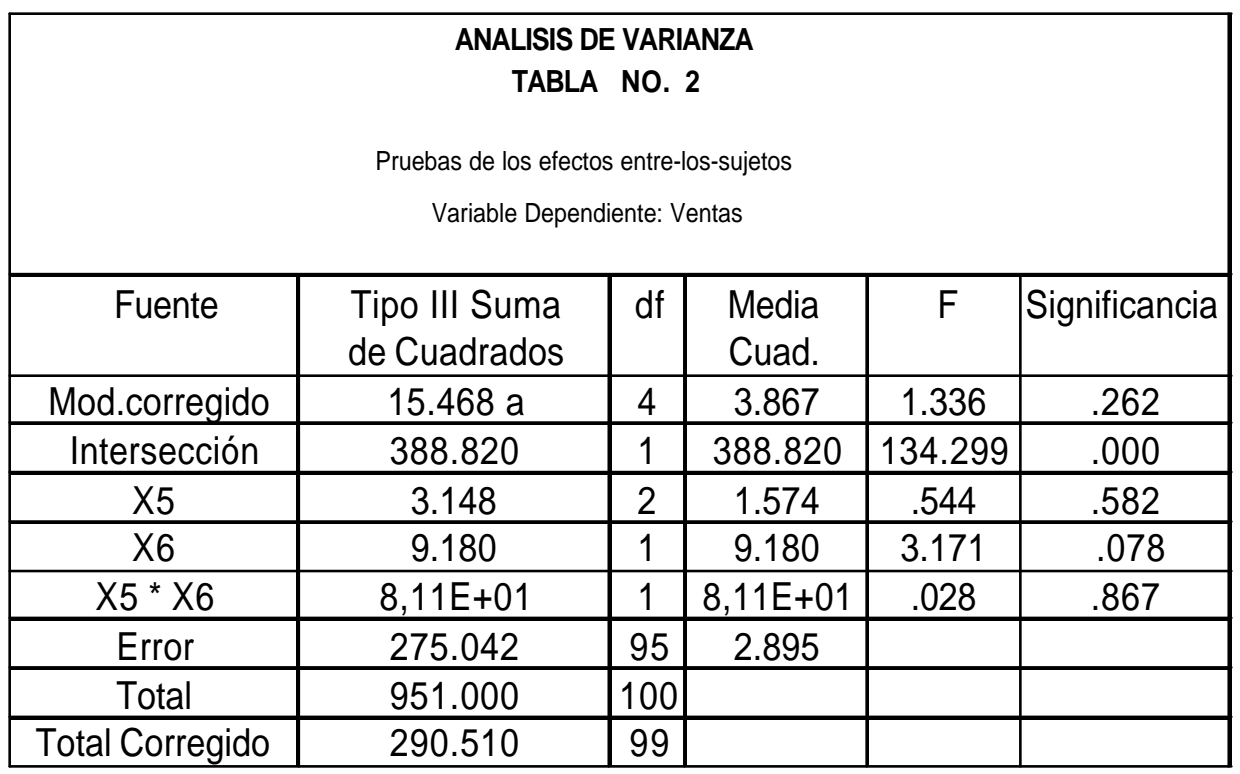

a. $\mathrm{R}$ Cuadrada $=.053$ (R Cuadrada Ajustada $=.013$ )

X5 = Estructura Organizacional $\quad$ X6 $=$ Complejidad

\section{Conclusiones}

El proceso de administración estratégica revisado en este estudio, es una herramienta moderna, práctica y sencilla para la adecuada gestión de un negocio. Actualmente no ha perdido su validez y su vigencia. Se puede considerar que sigue a la vanguardia en los procesos de mejoramiento institucional. La teoría de sistemas del biólogo Ludwig von Bertalanffy es muy útil para ser aplicada a los negocios actuales. Cuando es integrada a la administración estratégica, cobra un valor agregado su provecho en la aplicación del proceso estratégico.

El enfoque situacional, revisado también en este trabajo, e integrado a las dos escuelas anteriores del pensamiento administrativo, complementa de manera conveniente, las herramientas que necesita aplicar el gerente a un 
sistema de trabajo para hacerlo eficiente y poder competir en el mundo actual. El paradigma de estrategia y estructura (la estructura sigue a la estrategia) de Alfred Chandler presenta una yunta de conceptos que por muchos años se han esgrimido en la literatura administrativa y se han utilizado con éxito en la práctica gerencial, en el diseño de las organizaciones. Este modelo sigue siendo válido en la actualidad y se evidenció su aplicación de acuerdo a los hallazgos encontrados en este estudio. En las 100 empresas investigadas en el noreste de México se observó y comprobó que aunque las empresas se organicen de acuerdo al concepto de Chandler, su desempeño final no varía de acuerdo a esta concepción.

La autenticidad del paradigma de la relación extendida de estrategiaestructura-equifinalidad, entre las 100 empresas encuestadas, pudo evidenciarse con la prueba empírica que se hizo con la información obtenida. El principio de equifinalidad de von Bertalanffy, planteado inicialmente para analizar y comprender a los seres vivos (sistemas abiertos), tiene una gran aplicación a la administración presente. La prueba empírica de esta disertación demostró la certeza de la afirmación de que existe un diseño organizacional determinado y específico para cierto tipo de complejidad de una estrategia establecida. Asimismo, no importa, o no es necesario, que se presente esta relación para que se puedan obtener resultados exitosos en una empresa. Esto es, varias combinaciones (distintas) de estrategia y de estructura pueden resultar en un "fin igual" o equifinal.

Si el paradigma de equifinalidad se integra a la administración estratégica moderna, brinda, no solo las consideraciones de entender a la empresa como un sistema abierto y contingente, sino la oportunidad de conceptuar una nueva cosmovisión de la administración, al considerarla como de naturaleza, no solo estratégica, sino sistemática y situacional, en un solo punto de vista 0 enfoque. Si se agregan los conceptos de la Teoría del Caos a lo tratado en este documento, se puede concluir también, que no obstante que los sistemas orgánicos posean estrategias y estructuras ordenadas, disciplinadas, planeadas y organizadas, los resultados en el futuro, a largo plazo, podrán ser no medibles 0 infructuosos por lo incontrolable de las variables de los sistemas abiertos. Esto, que lo establece la Teoría del Caos dentro del enfoque de sistemas, no obstante, "podrá tender" hacia un resultado de beneficio, exitoso o de rendimiento por la naturaleza de la vida misma. La vida busca a la vida misma y la tendencia parece ser hacia la evolución constante, aunque se atraviese por etapas aparentemente infructuosas. Esta explicación caería dentro del terreno de la holística en el campo filosófico. 
Analizar el por qué de la certeza del paradigma probado en este estudio, de estrategia-estructura-equifinalidad constituye la "reserva de significados latentes", de este estudio, interpretando la aplicación del concepto del filósofo Raúl Gutiérrez Sáenz. Esta pregunta puede ser planteada más adelante, en otra exploración que genere estudios futuros. Por lo pronto, hoy se ha quedado en el tintero para posteriores investigaciones.

\section{Referencias}

Ashmos, D.P. y G.P. Huber. 1987. The Systems Paradigm in Organization Theory: Correcting the Record and Suggesting the Future. Academy of Management Review. 12: 607-621.

Bettis, R. A., y C. K. Prahalad. 1995. The dominant logic: Retrospective and Extension. Strategic Management Journal. 16: 5-14.

Burns, J. S. 2002. Chaos theory and leadership studies: Exploring uncharted seas. Journal of Leadership \& Organizational Studies. Flint Fall. 9(I2): 42.

Burns, T. y G. M. Stalker. 1961. The Management of Innovation. London. Tavistock Publications.

Certo, S.C. 2001. Administración Moderna, 8a. ed. Bogotá, Colombia: Pearson Educación de Colombia Ltda.

Chandler, A.D., Jr. 1962. Strategy and Structure. Cambridge, M.A. MIT Press.

Chennai, B. 2003. Of Chaos and Complexity. Businessline: 1.

DeGrauwe, P., H. Dewatcher y M. Embrects. 1993. Exchange rate theory: chaotic models of foreign exchange markets. Blackwell, Oxford, UK.

Deshpande, R. y J.U. Farley. 1998. Measuring Market Orientation: A Generalization and Synthesis. Journal of Market Focused Management 2: 213-232.

Dessler, G. 1979. Organización y Administración: Un Enfoque Situacional. Cali, Colombia. Prentice-Hall International.

Dill, W.R. 1958. Environment as an Influence on Managerial Autonomy. Administrative Science Quarterly 2: 409-443.

Doty, D.H., W.H. Glick y G.P. Huber. 1993. Fit, Equifinality, and Organizational Effectiveness: A Test of Two Configurational Theories. Academy of Management Journal 36: 1196-1250.

Drago, A.W., 1998. Mintzberg's Pentagon and Organisation Positioning. Management Research News 21(4/5): 30.

Galbraith, J.R. 1973. Designing Complex Organizations. Reading, M.A. Addison-Wesley.

Gleick, J. 1987. Chaos Making a New Science. N.Y. Penguin Books.

Gutiérrez, S. R. 1992. Introducción a la Filosofía. México: Editorial Esfinge, S.A. de C.V.

Gresov, C. y R. Drazin. 1997. Equifinality: Functional Equivalence in Organizational Design. Academy of Management Review. 22: 403-438.

Gurpreet, D. yJ. Ward. 2002. Chaos Theory as a Framework for Studying Information Systems. Information Resources Management Journal. 15(2): 5-9 . 
Hrebeniak, L., y W. Joyce. 1985. Organizational Adaptation: Strategic Choice and Environmental Determinism. Administrative Science Quarterly. 30: 336-349.

Hopkins, T. 1937. Integration: Its Meaning and Application. N. Y. Appleton-Century-Crofts.

Jennings, D.F. y S.L. Seaman. 1994. "High and Low Levels of Organizational Adaptation: An Empirical Analysis of Strategy, Structure, and Performance." Strategic Management Journal 15: 459-475.

Johnson, G. y K. Scholes. 2001. Dirección Estratégica, 5a. Ed. Madrid, España: Pearson Educación S.A.

Katz, D. y R.L. Kahn. 1966. The Social Psychology of Organizations. N.Y. John Wiley \& Sons.

1978. The Social Psychology of Organizations. N.Y: John Wiley \& Sons.

Kauffman, S. 1995. Laws of Self Organization. Oxford: (i) Oxford University Press.

Lawrence, P.R. y J.W. Lorsch. 1969. Organizations and Environments. Homewood, IL: Richard D. Irwin.

Lorsch, J.W. y J.J. Morse. 1974. Organizations and Their Members: A Contingency Approach. N.Y. Harper and Row.

Matsuno, K. y J.T. Mentzer. 2000. The Effects of Strategic Type on the Market OrientationPerformance Relationship. Journal of Marketing. 64: 1-16.

Meyer, A. D., A. S. Tsui y C. R. Hinings. 1993. Configurational Approaches to Organizational Analysis. Academy of Management Journal. 36: 1175-1195.

Miles, R.E. y C.C. Snow. 1978. Organizational Strategy, Structure and Process. N.Y. McGraw-Hill.

__ A.D. Meyer y H.J. Coleman Jr. 1978. Organizational Strategy, Structure, and Process. Academy of Management Review 12: 607-621.

Miller, D. 1987. The genesis of configuration. Journal of Management Studies. 12: 686701.

Mintzberg, H. T. 1979. The Structuring of Organizations. Englewood Cliffs, N.J. Prentice Hall.

1993. Structure in Fives: Designing Effective Organizations, 2nd Ed., Prentice-Hall. Englewood Cliffs, N.J.

Nadler, D. A., y M. L. Tushman. 1988. Strategic Organization Design. Glenwood, I.L. Scott.

Peters, E. 1991. Chaos And Order in Capital Markets. New York: John Wiley \& Sons.

Porter, M.E. 1980. Competitive Strategy. N.Y. Free Press.

Prichett, P. y R. Pound. 2001. The Stress of Organizational Change. Piano, Texas: Pritchett Rummler-Brache.

Pugh, D.S., D.J. Hickson, C.R. Hinnings y C. Turner. 1969. The Context of Organizational Structures. Administrative Science Quarterly. 14: 91-114.

Radzicki, M. J. 1990. Institutional Dynamics, Deterministic Chaos, and Self-organizing Systems. Journal of Economic Issues. 24,1,57-102.

Scott, W. R. 1992. Organizations: Rational, Natural, and Open Systems, 3rd Ed. Englewood Cliffs, N.J. Prentice-Hall.

Stacey, R. D. 1991. The Chaos Frontier - Creative Strategic Control for Business, Butterworth-Heinemann, Oxford. 
Stoner, J. A., F. R. E. Freeman y D. R. Gilbert, Jr. 1996. Administración. Prentice Hall Hispanoamericana, S. A.

Van de Ven, A.H. y R. Drazin. 1985. The Concept of Fit in Contingency Theory. Research in Organizational Behavior, Eds B.M. Staw and L.L. Cummings. Greenwich, C.T. JAI Press: 333-375.

Venkatraman, N. y J. E. Prescott. 1990. Environment-structure Coalignment: An Empirical Test of its Performance Implications. Strategic Management Journal. 11: 1-23.

Von Bertalanffy, L. 1930. Kritische Theorie der Formbildung. Vienna. Schaltz Publishing. 1960. General Systems Theory. New York, N.Y. George Braziller. 1968. General System Theory. New Cork, N.Y. Braziller.

Wechsler, J. 1998. Managed Care Firms are Kicking Butts. Managed Healthcare 8(4): 3236.

www.cyberspace.com/ building/ofc_21clidhock.html Hock, D.W. (1996), The Chaordic Organization: out of Control and Into Order.

http://www.deguate.com/infocentros/gerencia/glosario/a.htm

http://www.uned.es/master-seguros/consultas/consultas_2003-04.htm 